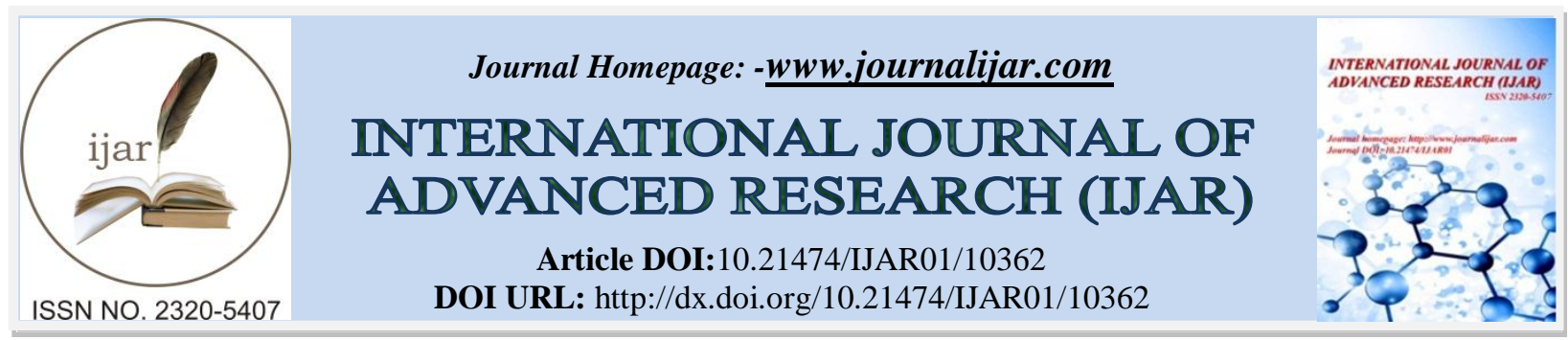

RESEARCH ARTICLE

\title{
STRATEGIE DE DEPLOIEMENT DES INSTITUTIONS FINANCIERES PARTICIPATIVES AU MAROC «CAS DU LINKING BANKING»
}

\section{Souad Merimi ${ }^{1}$ and Kaoutar El Menzhi ${ }^{2}$}

1. Université Mohamed V, Faculté des Sciences Juridiques Economiques et Sociales, Souissi, Rabat.

2. Laboratoire de Recherche en Management des Organisations, Droit des Affaires et Développement Durable.

\section{Manuscript Info}

………………….

Manuscript History

Received: 30 November 2019

Final Accepted: 31 December 2019

Published: January 2020

Key words:-

Finance, Islam, Principes, Prescriptions, Règles De La Jurisprudence, Finalités

De La Charia, Pensée Linéaire, Pensée

Complexe, Finance Éthique,

Management, Stratégie, Social,

Entrepreneuriat, Investissements,

Charia, Investissements Et Finance

Capitaliste

\section{Abstract}

Dans les quatre coins du globe, et depuis la crise financière de 2008 , les principes de la finance islamique font l'objet d'une attention accrue de la part des spécialistes du domaine. La première revue de la littérature montre que le sujet est appréhendé de manière linéaire et construite, dans une large mesure, en reflet inversé de la finance globale sans distinguer le fondamental du secondaire, le général du spécifique et du partiel. L'objectif de cet article est de présenter une première revue littérature permettant d'aborder le sujet de manière rigoureuse, sous le prisme des systèmes complexes, via une approche conceptuelle puisé dans le corpus méthodologique des sciences religieuses islamiques classiques. Au final, cette approche permet de distinguer les principes qui constituent des prescriptions de ceux qui constituent des normes, les principes qui constituent des prescriptions fondamentales de ceux qui constituent des prescriptions secondaires, les principes qui sont liés à une finalité générale de ceux qui sont liés à des finalités spécifiques.

La finance participative «islamique» de nos jours subit une grande influence de la finance conventionnelle, vu que cette dernière s'exprime directement dans la richesse, sa régulation et sa distribution dans le temps, différemment aux principes de la finance participative «islamique » qui rajoute d'autres principes. En dépit du concept de l'interdiction de l'intérêt «usure, riba » la finance participative «islamique» traite en ajout au développement de la richesse, le développement socio-économique et développement durable. De ce fait, la prise en considération de ces facteurs pourra être un facteur clé de succès très important dans le développement de la population.

Copy Right, IJAR, 2020,. All rights reserved.

\section{Introduction:-}

La finance se définit comme une activité de service. Elle a pour fonction de garantir la fluidité des transactions indispensables à l'activité économique et de permettre une utilisation optimale des capitaux disponibles dans l'économie (Bari et Radi, 2011). C'est pour cette raison qu'elle rassemble toutes les activités qui organisent le financement des agents économiques ayant des besoins de capitaux tels que les entreprises.

Suite à la crise financière qu'a connue le monde de la finance en 2007, les recherches se sont accentuées sur une finance qui ne soit pas centrée principalement sur le profit. De ce fait, les discours cumulant depuis sur les principes 
de la finance participative, construit en reflet inversé de la finance globale, affirme et célèbre la suprématie des principes moraux de l'Islam sur les décombres du capitalisme financier. Pourtant, la plupart des principes énumérés ont existé tout au long de l'histoire humaine dans différentes sociétés. La valeur de ces principes n'est donc pas uniquement fonction de leurs valeurs intrinsèques pondérées par leurs coefficients respectifs, ni de leurs juxtapositions, ni même de leurs interactions et rétroactions, aussi importantes soient-elles, mais de leur adossement au référentiel méthodologique des sciences juridiques islamiques classiques pour former des systèmes dynamiques et opérationnel, prenant en compte les dimensions spatiales et temporelles, ainsi que le patrimoine immatériel.

Toutefois, les principes d'un modèle économique et financier islamique sont révélés depuis longtemps dans les écrits théologique des écrivains musulmans, par contre leur éruption moderne et leur prise en main dans les pays du Moyen Orient et dans les pays de l'Asie et du Sud Est a été revue après l'indépendance des pays islamiques.

Sauf que le développement de ces institutions n'a pu suivre la même cadence dans les quatre coins du monde et principalement au Maroc. En effet, les pouvoirs publics marocains se sont attachés, dès les années 90, à moderniser le système financier national afin d'accroître son efficacité et d'améliorer son attractivité sur le plan international. Le secteur financier a ainsi connu une profonde mutation. Des réformes ont été engagées afin de doter le pays d'un système à même de mobiliser l'épargne de manière efficace et de l'allouer avec efficience au financement de l'économie. Ces réformes visaient par ailleurs à faire converger la règlementation marocaine en la matière vers les standards internationaux. De ce fait, depuis Octobre 2007, le Maroc et pour la première fois depuis l'indépendance, que la Bank Centrale «Bank Al-maghrib» autorise la commercialisation de nouveaux produits bancaires dits «produits alternatifs ou participatifs ». Donc un nouvel élan de la finance voit le jour au Maroc et pose une grande question.

Ce travail aura pour objectif la revue littérature des différentes rédactions touchant le développement de la finance participative au Maroc et particulièrement et la mise en place d'un modèle économique faisable pour la réussite de ces institutions sur le marché marocain, voir le développement le modèle de « Fintech au Maroc ou Linking Banking ».

\section{Problématique:}

Une situation problématique fondée sur de nombreuses interrogations qui tourne autour de la stratégie de déploiement des institutions financières participatives au Maroc.

De ce fait, ce projet compte apporter un éclairage sur les institutions financières participatives et projette de poser les jalons nécessaires à l'élaboration d'une stratégie de leur déploiement.

La combinaison de ces deux composantes dans la création d'une dynamique économique créatrice de la richesse en intégrant une dimension sociale et solidaire en tant que référence émergente pour appuyer la croissance économique marocaine, et susciter l'esprit entrepreneurial.

En vue d'interpeler les composantes de la stratégie en question de manière à y apporter une évaluation objective à même d'asseoir une plateforme solide et un palier favorable à l'atteinte des résultats escomptés, je laisse surgir dans le champ de ma problématique une grande question:

Quelles sont les limites phares du système financier des institutions de financement au Maroc ? et par quels moyens les institutions financières participatives vont-elles répondre aux manquements du système financier actuel et quels axes faut-il développer pour concevoir et mettre en œuvre une stratégie de déploiement des institutions précitées?

\section{Hypothèses de départ à vérifier:}

1. Hypothèse 1 : Ce modèle d'agir financier est générateur de revenus, créateur de l'emploi et de la richesse ;

2. Hypothèse $2:$ Ces institutions financières et participatives se présentent comme un moyen d'améliorer le climat d'affaire venant en réponse à la nécessité de développer l'entrepreneuriat au Maroc;

3. Hypothèse 3 : Ces organisations économiques favorisent la promotion du bien-être collectif et le développement communautaire ;

4. Hypothèse $4:$ La multiplicité des intervenants des acteurs du système financier conventionnel augmente sa rigidité en action et pénalise le consommateur final ;

5. Hypothèse 5: Ces luttes contre la précarité par des actions empreintes de solidarité visent à démocratiser l'économie par la lutte contre l'exclusion; 


\section{Etat de l'art:}

La finance islamique tire ses origines de la quête du savoir dans la péninsule arabique, se présente comme une forme d'intermédiation qui s'appuie sur les préceptes d'une religion qu'est l'Islam, (Dhafer SAIDANE, op.cit, p12).

Malgré l'historique de ce concept, le renouveau d'intérêt pour cette forme particulière de finance est, lui, relativement récent. La finance islamique dite moderne est réellement née dans les années 1960.

Les premières prémices de la finance islamique date de l'âge d'or de l'islam entre le VIIe et XVIe siècle, c'est-àdire à partir du début de la révélation de loi divine (le Coran) par Dieu au prophète Mohamed (PSSL).

Suite à cette période d'âge d'or, et dans les années soixante-dix, apparait le système de finance islamique moderne stimulé principalement par un regain de vitalité de la religion musulmane et par l'importance des ressources financières de certains pays musulmans.

Figure 1:- Evolution historique des institutions de la Finance Islamique.

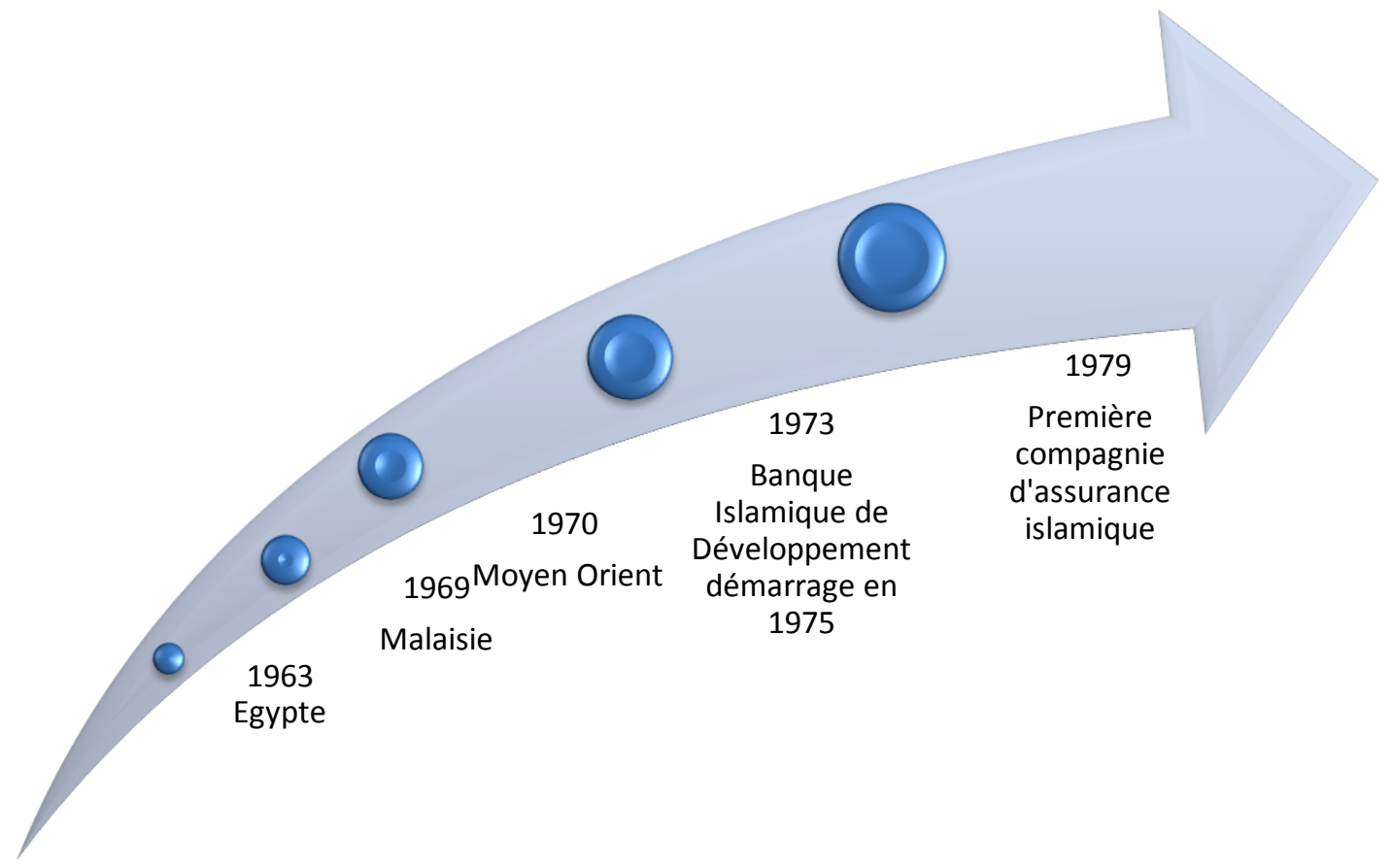

La finance participative « islamique » est une démonstration de la finance qui entend se mettre à la disposition de l'économie réelle et la servir. En reliant la finance à l'éthique de l'économie islamique, il apparaît que la focalisation sur la prohibition de l'intérêt est réductrice. En effet, l'absence d'intérêt n'est que la conséquence logique d'une dynamique d'accroissement pérenne ancrée dans l'économie réelle. L’économie islamique est fondée sur un paradigme dont l'objectif primordial est la justice socio-économique.

En effet, l'éthique issue de l'économie islamique vise à instaurer une société où les ressources sont utilisées de façon optimale et le mode de rémunération du financement est étroitement lié aux résultats de l'activité financée. Par sa définition, cette finance recouvre l'ensemble des techniques financières et juridiques permettant le financement de biens et services conformément aux exigences de la loi coranique (la charia). Ces techniques dont les valeurs éthiques se distinguent dans un contexte de crise financière, représente une manne financière considérable.

La croissance économique souhaitée est donc celle qui se réalise sûrement à l'aide de la finance, mais aussi à travers le comportement que cette éthique prône. À ce propos, j'avance que la portée réelle de cette éthique ne pourrait être saisie en se focalisant uniquement sur la prohibition de l'intérêt. Ceci permet de comprendre pourquoi en finance islamique, aucun contrat n'est légitime s'il permet à l'argent de créer de l'argent sans l'association du capital physique et du travail dans une activité productive bénéfique à la société. Ce qui fait la particularité des types de contrats 
proposés dans le domaine de la finance islamique qui repose sur le principe du partage de gain et le risque de la perte est toujours mesuré. Donc, la croissance économique et l'accroissement de la richesse matérielle sont parfaitement compatibles avec la vision islamique du monde, à condition que cette recherche d'une richesse matérielle respecte l'éthique islamique. Cependant, tout en admettant que le développement matériel et l'accroissement de la richesse et des revenus sont souhaitables et nécessaires à la satisfaction des besoins essentiels, la vision islamique du monde ne peut s'accommoder d'une réduction du développement à cette seule dimension matérielle.

Ainsi, pour réaliser le développement et stimuler la croissance économique, cette finance ne devra pas se limiter à des solutions techniques et des mécanismes pratiques. L’apport de ces solutions est très limité puisque les capitaux mobilisés par ces techniques peuvent être investis moyennant d'autres mécanismes, qui peuvent ne pas être licites mais plus efficaces pour réaliser des gains. L'accent est mis ici sur l'association entre l'investissement et la nécessité d'observer une conduite éthique.

L'économie et la finance islamiques se situent encore loin de l'objectif escompté, puisque leur contribution à la résolution des problèmes sociaux et économiques, au moins pour les sociétés musulmanes, est très faible (Chapra, 1996). Ce système est appelé d'abord à relever un certain nombre de défis relatifs à la pratique financière islamique à partir d'une analyse critique de la finance capitaliste.

L'intégration de la finance islamique dans le système financier national et international est conditionnée par l'harmonisation des avis juridiques et la bonne interaction des règles de la charia islamique avec les systèmes juridiques et le droit civil mis en place (Iman et Radi, 2011). Il semble donc que l'un des plus grands défis de la finance islamique consiste à créer un cadre pour la gestion, le contrôle et la réglementation des institutions financières participatives.

En outre, par principe ou par opportunisme, l'engouement pour la finance islamique peut contribuer à son positionnement stratégique en tant qu'alternative crédible et viable à l'échelle planétaire (Elmeziane, 2013). Toutefois, selon l'agence Moody's, la finance islamique ne gagnera ses lettres de noblesse et ne pourra accéder à une forme de reconnaissance internationale qu'à un certain nombre de conditions comme la transparence et la gouvernance.

L'économie Islamique regroupe des sujets aussi variés que la fiscalité, les dépenses publiques, l'intérêt, la propriété financière, les ressources naturelles, le taux de salaire, les promoteurs de l'islamisation de l'économie, jettent leur dévolu sur les systèmes financiers considérés comme plus accessibles pour deux raisons:

1. L'évidence de l'identité de l'intérêt « Riba » et la prohibition de celui-ci parmi les populations musulmanes ;

2. L'éloignement de proximité entre ces population et système financier conventionnel, et dans ce sens, la mise en place de ce système dans une économie non islamisé à cent pour cent est jugée couteux par contre dans des économies telles que «Pakistan, Iran, Soudan » ce système réussi chose qui est due à l'islamisation de leurs économie.

Il convient donc à ces techniques, relevant de la finance islamique, d'être nourri par une éthique qui fasse que le gain et le bénéfice ne soient pas les seules motivations des opérateurs économiques. Ainsi, la philosophie générale d'objectifs supérieurs et la conscience des finalités sociales doivent accompagner l'application de ces techniques. En constituant une diversification opportune des sources de financement pour les entreprises et tout le tissu industriel, il convient à la finance participative «islamique » de prendre sa place et de jouer pleinement son rôle en étant une finance d'éthique.

Dans ce système économique et financier, la finance islamique repose sur l'intégration des dimensions sociales, environnementales, gouvernementales dans l'analyse de l'investissement à accompagner. Cela illustre le principe de la finance éthique qui mène sur le chemin de la création d'une économie et d'un finance reposant sur l'éthique, ce qui va permettre la création des investissements et / ou entreprises qui veilleront à progresser suivant une gestion éthique. 
Figure 2:- Dimension de l'économie islamique ${ }^{2}$

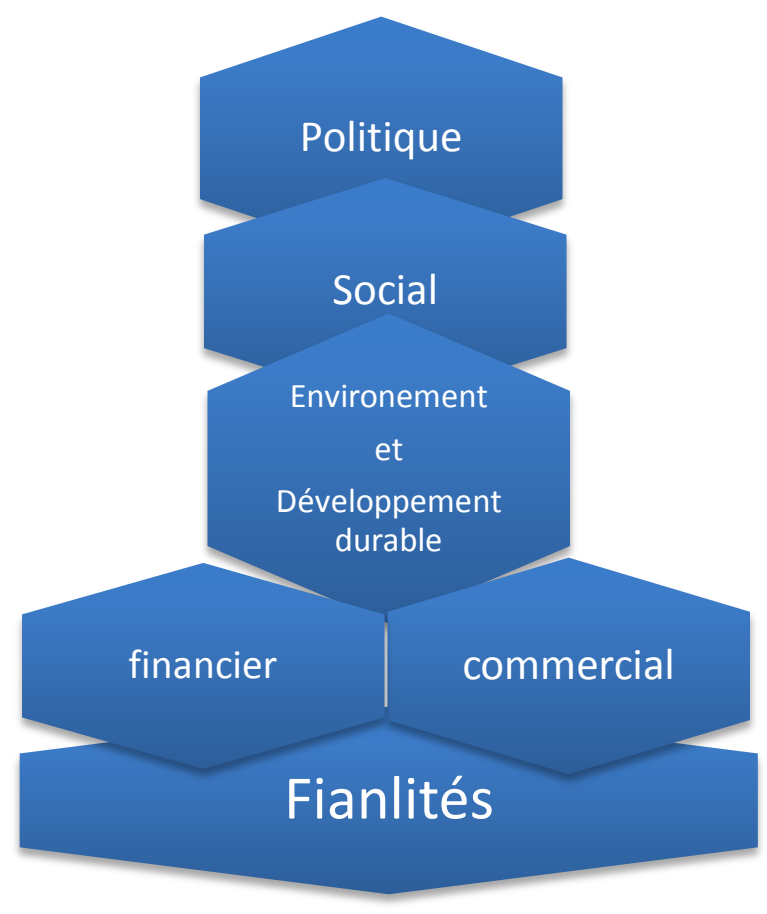

2 Abderrahmane LAHLOU, Economie et Finance en Islam, Une éthique pour stabiliser l'économie et recadrer la finance, 2015

\section{La finalité en théorie de l'économie et la finance islamique repose sur :}

1. Références de l'économie en islam est la chariaa appelé aussi Maqassid,

2. Finalités économiques visent plutôt à assurer des conditions de vie décente et inclusive pour la communauté de la population

3. Economie islamique est fondée sur un paradigme dont l'objectif primordial est la justice socioéconomique « à la différence du paradigme séculier du marché, le bien être humain ne dépend pas essentiellement de la maximisation de la richesse et de la consommation. Il exige une satisfaction équilibrée des besoins tant matériels que spirituels de la personnalité humaine. (Umer Chapra 1996).

Il est évident que cette finance doit jouer un rôle grandissant pour rendre ces stratégies faisables par les solutions techniques qu'elle propose et la qualité des interactions économiques qu'elle permet. À cet égard, la mission de cette finance islamique est de contrecarrer les éléments qui conduisent tout naturellement à la perte d'autonomie, à la marginalisation sociale et à l'exclusion. Cela se voit d'une manière plus explicite dans le fait d'éviter le surendettement et d'occulter les charges financières inutiles.

Cela nécessite une prise de conscience profonde afin d'établir des structures sociales qui viennent en aide au processus engagé. Une telle conscience est susceptible de propulser le système économique vers plus de justice et la population vers plus de bien-être. En s'adonnant à ce processus de prise de conscience, la tâche consiste à promouvoir l'application de l'éthique à la finance et, par la suite, à l'économie en allant des retombées positives sur la population.

\section{Conclusion:-}

Le développement économique d'une nation constitue un but très recherché par les différents systèmes économiques dans le monde, mais elle n'est pas toujours dans l'intérêt de l'homme et pourra avoir un impact néfaste sur le développement de la population concernée. 
Ce qui réapparait dans la présentation du principe de la finance participative découlant du principe du partage de gains et du risque parcouru, et cela réside dans le principe du partenariat et non seulement un simple emprunt ou coffre de dépôt. De ce fait, dans la stratégie de la finance participative, il est prioritaire de mettre le point sur le revenu du capital considéré dans l'évaluation des projets par les banques islamiques ainsi que l'intérêt social.

Donc l'ouverture des systèmes économiques et financiers à la finance participative « islamique » ouvrira ainsi la voie à la mobilisation des ressources disponibles dans la société concernée et à la réalisation des projets d'investissement qui ne pouvaient voir le jour autrement. Cela mobilisera aussi une catégorie qui était stérile vis-àvis de la mobilisation de ses fonds dans l'investissement de la finance conventionnelle, et qui aura enfin l'occasion de s'exprimer et d'être un acteur actif dans le développement des affaires et de l'économie nationale et internationale en créant un social marquant.

\section{Bibliographie:-}

1. Belabes, a. ; (2013) «éthique, normes et finance: de quoi parlons-nous? », études en économie islamique, séminaire à l'université paris 1 panthéon-sorbonne, le 9 févr. 2013 ;

2. Chapra umer; (2009) «the global financial crisis: can islamic finance help? », academic article, newhorizon january-march 2009 p. 21.

3. Kettani malika; «une banque originale la banque islamique», dar al-kotob al-ilmiyah, beyrouth, liban3ème édition 2014,

4. Lahlou abderrahmane, économie et finance en islam. Une éthique pour stabiliser l'économie et recadrer les finances, éd. Al madariss, avril 2015,

5. Wadi mzid - directeur d'agence à la banque zitouna, tunisie; la finance islamique : principes fondamentaux et apports potentiels dans le financement de la croissance et du développement,

6. El hazzaouni youssef, finance islamique : fondements, mécanismes et apports broché, 1 janvier 2012.

7. La finance islamique est-elle un moyen de régulation ?, imane bari et bouchra radi, au-delà de la crise. Article publié en 2011

8. «la finance islamique : un levier à exploiter pour le développement et la lutte contre les inégalités », article de la banque mondiale, février 2017. 